\title{
Tracking with the LHCb Spectrometer: Detector Performance and Track Reconstruction *
}

\author{
N. Tuning \\ on behalf of the LHCb collaboration \\ NIKHEF, Kruislaan 409, 1098 SJ Amsterdam, The Netherlands
}

\begin{abstract}
The $\mathrm{LHCb}$ experiment aims to measure $\mathrm{CP}$ violation and rare $B$-decays. For this, a tracking system is constructed consisting of a silicon micro-strip vertex locator close to the interaction point, and tracking detectors around a dipole magnet. The resulting tracking performance is estimated from simulation to yield $95 \%$ efficiency. The momentum and impact parameter resolutions vary between $0.35 \%$ and $0.5 \%$, and $20 \mu \mathrm{m}$ and $160 \mu \mathrm{m}$, respectively.
\end{abstract}

Key words: LHC, LHCb, tracking, gas detectors

\section{The LHCb Spectrometer}

The $\mathrm{LHCb}$ experiment is a single arm spectrometer, designed to study $B$ decays at the Large Hadron Collider ( $\mathrm{LHC}$ ), covering the range $1.8<\eta<4.9$ in rapidity. $\mathrm{CP}$ violation parameters and rare decays are sensitive to physics beyond the Standard Model. A good tracking system is essential to determine the proper lifetime of the $B$-mesons and to accurately determine the momentum of its decay particles. The tracking system is divided in a silicon detector close to the interaction region (the vertex locator, VELO), a dipole magnet, and a tracking system behind the magnet, see Fig. 1. The tracking system behind the magnet is divided in two parts: a silicon detector at high rapidity in the highest particle flux region, the Inner Tracker (IT), and a gaseous straw tube detector, the Outer Tracker (OT). In addition a silicon detector,

ॠ Presented at the Imaging2006 conference, Stockholm, Sweden 27-30 June 2006 

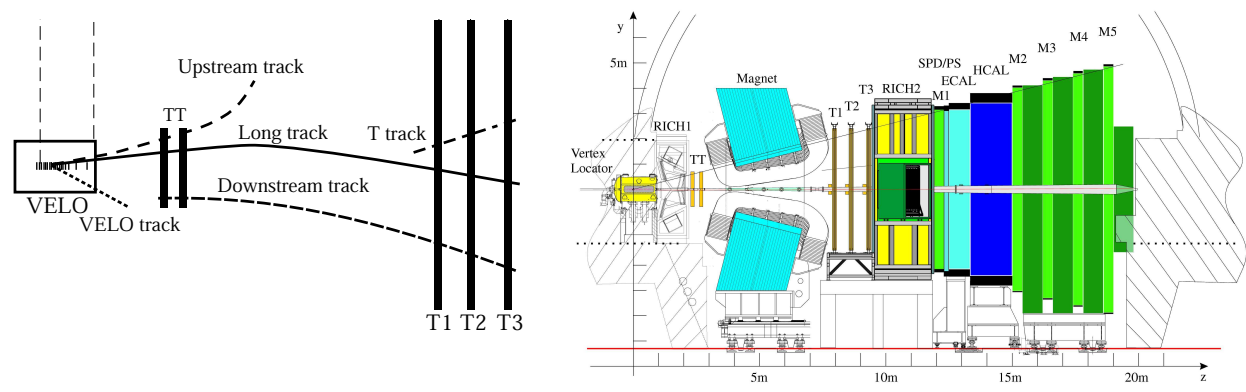

Fig. 1. A schematic picture of the LHCb detector.

the Trigger Tracker (TT), is placed in the fringe field before the dipole magnet. The detector systems will be described in Sec. 2, whereas the expected tracking performance will be discussed in Sec. 3 .

\section{Tracking Detectors}

The VELO [1], see Fig. 2(a), is positioned around the interaction point, consisting of $21 R$ - and $21 \phi$-measuring sensors on either side of the beamline, resulting in a total of 84 microstrip silicon sensors. The sensors are positioned as close as $8 \mathrm{~mm}$ to the beam, whereas they are retracted during LHC beam injection. The large inhomogeneous irradiation is expected to vary from 0.5 $10^{13} \mathrm{neq} / \mathrm{cm}^{2} /$ year on the outside of the sensors at a radius of $42 \mathrm{~mm}$, to about $1.310^{13} \mathrm{neq} / \mathrm{cm}^{2} /$ year closest to the beam. The strip pitch varies between 39 and $102 \mu \mathrm{m}$ to obtain the desired vertex and impact parameter resolutions. The TT [2] is placed in the fringe field before the spectrometer dipole magnet. The magnetic field deflects the charged particles sufficiently

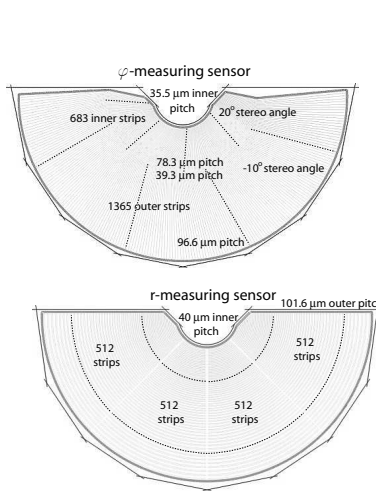

(a)

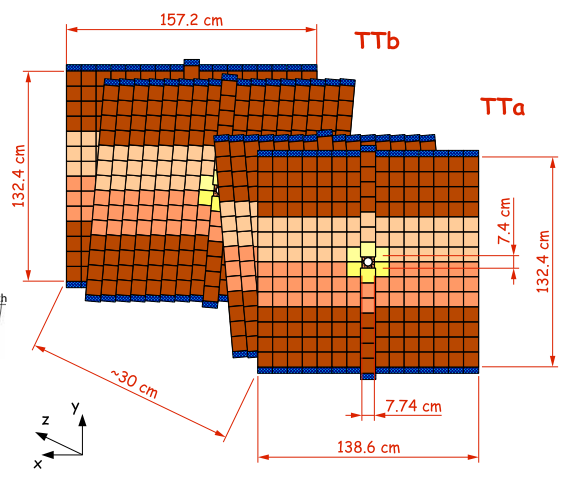

(b)

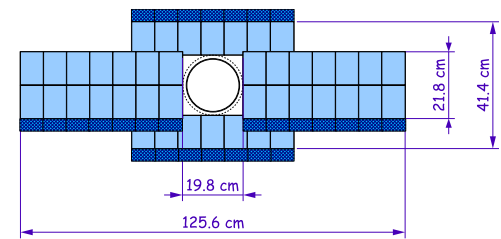

(c)

Fig. 2. Front view of the silicon detectors, (a) the Vertex Locator, (b) the Trigger Tracker and (c) the Inner Tracker. 


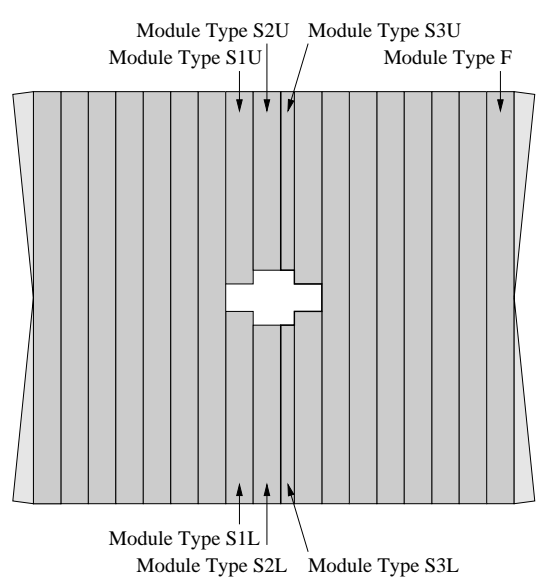

(a)

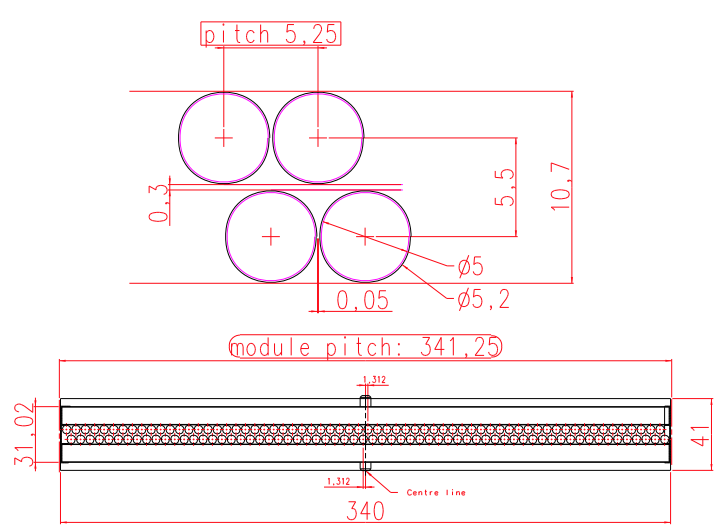

(b)

Fig. 3. The design of the Outer Tracker. (a) The front view of an entire tracker station. (b) One OT module consists of two mono-layers with 64 straws each.

to ensure a momentum resolution of approximately $d p / p \sim 30 \%$, which is used to select $B$-decay tracks in the trigger. The TT covers the full LHCb acceptance of $300(250) \mathrm{mrad}$ in $x(y)$, and is made of 4 layers of $183 \mu \mathrm{m}$ pitch slicon strip sensors of $9.6 \times 9.8 \mathrm{~cm}^{2}$. The use of thick silicon of $500 \mu \mathrm{m}$ compensates the lower signal-to-noise ratio due to the long kapton cables that connect the inner sensors to the front end readout.

The tracking system behind the dipole magnet consists of the IT [3] close to the beam, made of 12 layers of silicon sensors of $7.8 \times 11 \mathrm{~cm}^{2}$ with a strip pitch of $198 \mu \mathrm{m}$. The thickness of the two connected sensors on either side of the beam are $420 \mu \mathrm{m}$ thick, whereas the single sensors above and below the beampipe are only $310 \mu \mathrm{m}$ thick, limiting the total radiation length. The OT [4] covers an area of approximately $5 \times 6 \mathrm{~m}^{2}$ with 12 double layers of straw tubes. The straw tubes are $2.4 \mathrm{~m}$ long and $4.9 \mathrm{~mm}$ in diameter, and are filled with a gas mixture of $\mathrm{Ar}(70 \%)-\mathrm{CO}_{2}(30 \%)$. The anode is made of $25 \mu \mathrm{m}$ gold plated tungsten wire. Test beam experiments with a $6 \mathrm{GeV}$ electron beam at DESY [5] have shown that the required single hit resolution of $200 \mu \mathrm{m}$ is achieved. A single straw efficiency of larger than $98 \%$ was obtained at high voltage values as low as $1550 \mathrm{~V}$ and reasonable values for the amplifier threshold. The construction of all detectors is well under way and is partially installed in the LHCb detector.

\section{$3 \quad$ Tracking with $\mathrm{LHCb}$}

The resulting tracking performance is estimated from detailed Monte Carlo simulations [2]. First, straight line segments are constructed in the VELO. 

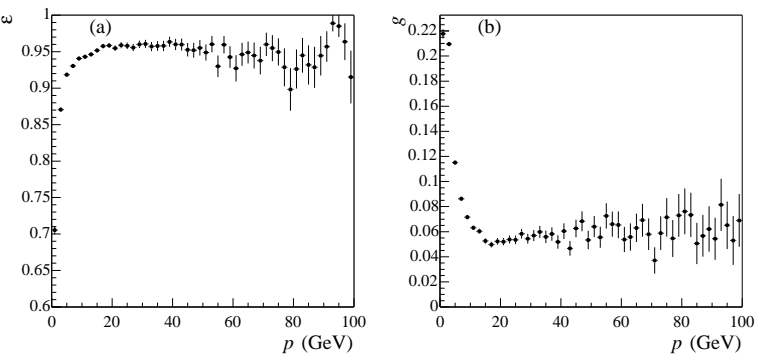

(a)
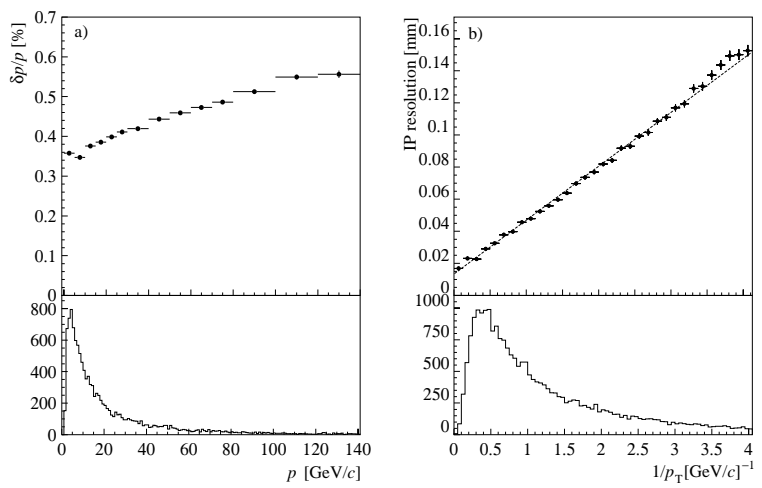

(b)

Fig. 4. (a) Efficiency and ghost rate of long tracks. (b) Momentum and impact parameter resolution of long tracks.

Subsequently, these segments are extrapolated through the magnet and the hits from the IT and OT are added. To obtain maximal efficiency, long tracks are also constructed by starting with track seeds in the tracking stations after which they are extrapolated back to the vertex. These long tracks are constructed with an efficiency of $95 \%$ and are of highest quality, see Fig. 4(a). The momentum and impact parameter resolutions vary between $0.35 \%$ and $0.5 \%$, and $20 \mu \mathrm{m}$ and $160 \mu \mathrm{m}$, respectively, see Fig. 4(b). Additionally, shorter tracks are reconstruced, see Fig. 1. VELO tracks with just VELO hits aid in the vertex reconstruction. Upstream tracks consist of VELO segments and TT hits, and improve the pattern recognition in the first Ring Imaging Cherenkov Counter (RICH1). Downstream tracks have hits in TT and the tracking stations behind the magnet and are needed for $K_{S}$ reconstruction. Finally, track seeds with hits in the tracking stations only help the RICH2 pattern recognition. Together, about 100 tracks per event are reconstructed in the $\mathrm{LHCb}$ experiment, of which about 38 are long tracks.

\section{References}

[1] LHCb Coll., P.R.Barbosa et al., Vertex Locator TDR, CERN/LHCC 2001-011

[2] LHCb Coll., R.Antunes Nobrega et al., LHCb Reoptimized Detector Design and Performance TDR, CERN/LHCC 2003-030

[3] LHCb Coll., A.Franca et al., Inner Tracker TDR, CERN/LHCC 2002-029

[4] LHCb Coll., P.R.Barbosa et al., Outer Tracker TDR, CERN/LHCC 2001-024

[5] G. van Apeldoorn et al., Beam Tests of Final Modules and Electronics of the LHCb Outer Tracker in 2005, LHCb 2005-076 